\title{
Mixed associations observed between advanced paternal age and psychiatric morbidity in offspring
}

\author{
Ranjith Ramasamy, Larry I Lipshultz \\ Department of Urology, Baylor College of Medicine, Houston, Texas, USA \\ Correspondence to Larry I Lipshultz; larryl@bcm.edu
}

\section{WHAT IS ALREADY KNOWN ON THIS TOPIC?}

Advanced maternal age has long been acknowledged as a risk factor for genetic disorders such as Down's syndrome. Owing to the large number of cell divisions that occur during spermatogenesis, we can speculate that advanced paternal age (APA) can contribute to an increased number of de novo mutations. ${ }^{1}$ Autosomal dominant diseases such as achondroplasia and multiple endocrine neoplasia syndrome are strongly associated with APA. However, the association between APA and complex disorders such as cancer and psychopathology is less clear.

\section{WHAT DOES THIS PAPER ADD?}

- This is the largest population-based investigation to date to address the association between advanced paternal age and psychiatric morbidity in the offspring. Previous studies have demonstrated inconsistent epidemiological findings on the association between psychiatric disorders and parental (both paternal and maternal) age. $^{2}$

- APA was associated with an increased risk for autism, psychosis and bipolar disorder and reduced risk of attention deficit hyperactivity disorder (ADHD), suicidal behaviour, substance abuse, failing grades in school, low-educational attainment and low 10.

- Sibling-comparison analysis demonstrated offspring of fathers older than 45 years were at significantly higher risk for autism, ADHD, psychosis, bipolar disorder, suicide attempts, substance abuse, failing grades and low-educational attainment, than offspring of fathers aged 20-24 years. Interestingly, the analyses demonstrated a dose-response relationship for increasing risk of mental health disorders with increasing paternal age.

\section{LIMITATIONS}

- Even though the sibling design obviates the need for data on parenting behaviours of older versus younger fathers, estimates can be severely biased by confounders not shared between the siblings.
- Diagnoses (except for ADHD and autism) relied on inpatient records. Potential cases of bipolar and other psychotic disorders managed in the outpatient setting could have been missed.

- The study included premature infants (aged 23-36 weeks), who are at greater risk for mental and intellectual disorders. ${ }^{3}$

\section{WHAT NEXT IN RESEARCH?}

The underlying genetic and molecular causes of why APA could contribute to increased psychiatric morbidity need to be determined.

\section{COULD THESE RESULTS CHANGE YOUR PRACTICES AND WHY?}

Yes, couples with an older male partner can be counselled regarding the small but increased risk of de novo mutations and psychiatric disorders. Nevertheless, the pregnancy should be treated as any other according to prenatal diagnosis guidelines. ${ }^{4}$ The clinical message is important; APA should not be a contraindication for conception. There are currently no screening or diagnostic test panels that specifically target those conditions that increase with paternal age.

Competing interests RR is an NIH K12 Scholar supported by a Male Reproductive Health Research Career, Development Physician-Scientist Award (HD073917-01) from the Eunice Kennedy Shriver National Institute of Child Health and Human Development Programme.

doi:10.1136/eb-2014-101891

\section{REFERENCES}

1. Kong A, Frigge ML, Masson G, et al. Rate of de novo mutations and the importance of father's age to disease risk. Nature 2012;488:471-5.

2. McGrath JJ, Petersen L, Agerbo E, et al. A comprehensive assessment of parental age and psychiatric disorders. JAMA Psychiatry 2014;71:301-9.

3. D'Onofrio BM, Class $Q A$, Rickert ME, et al. Preterm birth and mortality and morbidity: a population-based quasi-experimental study. JAMA Psychiatry 2013;70:1231-40.

4. Toriello HV, Meck JM; Professional Practice and Guidelines Committee. Statement on guidance for genetic counseling in advanced paternal age. Genet Med 2008;10:457-60.

ABSTRACT FROM: D'Onofrio BM, Rickert ME, Frans E, et al. Paternal age at childbearing and offspring psychiatric and academic morbidity. JAMA Psychiatry 2014;71:432-8.

Patients/participants All individuals $(n=2615081)$ born in Sweden between 1973 and 2001. Data obtained from births, deaths, migration, patient and other registers.

Setting Sweden.

Exposure Paternal age at childbearing (grouped into seven categories of 5 -year intervals, from 20 years or younger to older than 45 years).

Comparison Paternal age 20-24 years. A fixed-effects model compared paternal siblings to account for shared genetic and environmental factors, with additional adjustment for various other child and parental covariates. Follow-up period 2009, or date of death if prior to 2009.

\section{OUTCOMES}

Prevalence of morbidity Six indices of psychiatric morbidity were assessed (ICD criteria): autism spectrum disorder (ASD) and ADHD, psychosis, bipolar disorder, suicide attempt and substance use problem. Overall prevalence of psychiatric morbidity among children ranged from $0.27 \%$ to $3.04 \%$. Four indices of academic morbidity were assessed: failing grades, loweducational attainment (less than 10 years), high-educational attainment (more than 3 years postsecondary education) and low IO. Prevalence of academic morbidity ranged from $9.77 \%$ to $28.86 \%$.

Psychiatric morbidity Children of fathers aged 45 years or older were at increased risk of developing ASD ( $\mathrm{HR}=3.45,95 \%$ CI 1.62 to 7.33 ), $\mathrm{ADHD}$ (13.13, 6.85 to 25.16$)$, psychosis $(2.07,1.35$ to 3.20$)$, bipolar disorder (24.70, 12.12 to 50.31$)$, suicide attempt $(2.72,2.08$ to 3.56$)$ and substance use problems (2.44, CI 1.98 to 2.99).

Academic morbidity Children of fathers aged 45 years or older were at increased risk of failing a grade $(\mathrm{OR}=1.59,95 \%$ CI 1.37 to 1.85$)$ and loweducational attainment (1.70, CI 1.50 to 1.93$)$. 